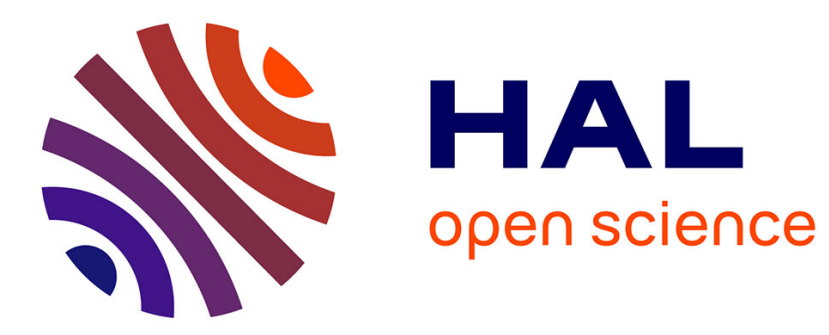

\title{
3D interaction technique to enhance telemanipulation tasks using virtual environment
}

\author{
Nassima Ouramdane, Frédéric Davesne, Samir Otmane, Malik Mallem
}

\section{To cite this version:}

Nassima Ouramdane, Frédéric Davesne, Samir Otmane, Malik Mallem. 3D interaction technique to enhance telemanipulation tasks using virtual environment. IROS 2006, Oct 2006, Beijing, China. pp.5201-5207, 10.1109/IROS.2006.281658 . hal-00339477

\section{HAL Id: hal-00339477 https://hal.science/hal-00339477}

Submitted on 29 Nov 2008

HAL is a multi-disciplinary open access archive for the deposit and dissemination of scientific research documents, whether they are published or not. The documents may come from teaching and research institutions in France or abroad, or from public or private research centers.
L'archive ouverte pluridisciplinaire HAL, est destinée au dépôt et à la diffusion de documents scientifiques de niveau recherche, publiés ou non, émanant des établissements d'enseignement et de recherche français ou étrangers, des laboratoires publics ou privés. 


\title{
3D interaction technique to enhance telemanipulation tasks using virtual environment
}

\author{
Nassima OURAMDANE \\ Frédéric DAVESNE \\ Samir OTMANE \\ Mallik MALLEM \\ IBISC Laboratory \\ University of Evry / CNRS FRE 2873 \\ 40, rue du Pelvoux, 91000 Evry, France \\ Email: \{nassima.ouramdane,frederic.davesne,samir.otmane,malik.mallem\}@ibisc.fr
}

\begin{abstract}
This paper gives preliminary results about the utilization of an interaction technique called FOLLOW-ME to fasten the selection task for teleoperation system. The implementation of an interaction between a user and a Virtual Environment (VE) in Virtual Reality (VR) may use various techniques. However, in the case of teleoperation, the interaction must be very precise and comfortable for the user. The model associated to the FOLLOWME technique splits the Virtual Environment into three zones in which a specific interaction model is used: a free manipulation zone, a scaled manipulation zone and a precise manipulation zone. Each one of the three zones is characterized by a specific interaction granularity. In the precise manipulation zone, we use the concept of virtual guides in order to assist the user to achieve his task. In this paper, our aim is to show that the FOLLOW-ME technique is well suited for selection in teleoperation tasks. To do this, we have first compared the FOLLOW-ME technique with classical interaction techniques in a virtual environment where different targets are situated at different depth and may move. The preliminary results show that our technique is more efficient than the classical Go-Go and Ray-casting techniques, in a sense that the task is more reproducible and easier to accomplish by the user. In a second stage, we use this result to design selection procedures for the ARITI tele-operation system and show that the use of FOLLOW-ME induces benefits for the user.
\end{abstract}

\section{INTRODUCTION}

Early Computer Assisted Teleoperation (CAT) architectures furnished to the operator many kind of assistances [4]. The tendency of these assistances was mainly focused on the operator interface improvement. In this context, several assistance strategies and informations concerning tasks were proposed. Nowadays, the CAT architectures benefit from a considerable boost due to virtual reality and augmented reality techniques which sets high standards in the human-machine interface [3]. There exists numerous reasons for using Mixed Reality (MR) for teleoperation such as, for instance, sensory feedback improvement. The basic control of task execution is carried out by superimposing the corresponding robot and its surroundings virtual model on the on line video feedback [6]. During the last decade, MR has been proved to be useful to improve performances of a user while this one is performing complex tasks. For example, the ARITI system helps the operator for teleoperation tasks of a remote robot [10].

In this paper, we address the issue of the efficiency of the interaction between the user and the distant world. We focus particularly on the interaction between the user and the virtual tool which movements are translated into the distant robot movements, for a selection task.

There are a lot of existing human-computer interaction techniques which attempt to solve the problem of grabbing and manipulating objects in VEs (see [1] for a state of the art about the 3D user interaction techniques). Among all the techniques, we think that the Go-Go technique [11] or the Ray-casting technique [7] may be well suited for selecting and manipulating remote objects, although they have been developed for interacting within immersive VEs. However, these two techniques are known to lake precision when the target to reach is far or small. But teleoperation tasks may have to be precise and easy to execute by the user.

The FOLLOW-ME technique [8] may be seen as an assistance to existing interaction techniques (Go-Go or Raycasting) in order to comply with both precision and easiness. Let's describe the idea behind the FOLLOW-ME technique. In today's life, if one wants to grab an object, his arm is firstly moving fast to the target and, when approaching, it slows down and then the hand uses a grabbing strategy to take the object. The different steps of the task may be characterized by different needs of precision (lower precision far from the target and higher precision near to the target). This induces the idea of granularity of interaction that is used in our model. What precisely interests us in the splitting of VE is the possibility to have both a wide zone in which the user interacts freely and realisticly with the virtual world and also limited zones near the objects to select or manipulate where there exists a strong assistance to the user that offers him precision and easiness to accomplish his task. The assistance may be materialized by visual cues in the VE or an active assistance to reach and grab an object. The FOLLOW-ME technique uses virtual guides [9] [13] for this active assistance.

In this article, we first try to specify the situations in which the FOLLOW-ME technique may bring benefits to the user. To do that, we have set a first set of experiments called "validation experiments" in a simple VE in which we measure the effectiveness of the assistance of the FOLLOW-ME technique to the classical Go-Go and Ray-casting techniques. In a second stage, we exploit the former results in order to design a proper selection procedure for the ARITI tele-operation system. This 
gives raise to a second set of experiments called "ARITI experiments".

In section II, we present a short review of related 3D interaction techniques which are commonly utilized in the context of VR. Section III gives some outlines about the the formal model of the FOLLOW-ME technique (see [8] for a complete description of the FOLLOW-ME model). Section IV gives the experimental protocol for the two sets of experiments. We use a formal measurement method inspired from the testbed evaluation method [2] in order to evaluate the performance of interaction of the classical and assisted techniques. Results are given in section $\mathrm{V}$. The first results show that our technique brings useful assistance to achieve a selection task.

\section{RELATED WORK}

Obtaining realistic interactions between user and a virtual world is the main issue of the majority of applications in Virtual Reality. Several common techniques for basic 3D tasks have mostly been developed in the context of Virtual Environment nowadays, but many of them are not useful in other types of 3D systems such as Mixed Environment (ME). There are four basic 3D interaction tasks that are found in most complex 3D applications: the Navigation task, the Selection task, the Manipulation task and the System Control task. In this paper, we'll be discussing 3D interaction technique for Selection tasks.

Selection is the specification of an object or a set of objects for some purpose. Manipulation refers to the specification of object properties (in general position and orientation). Selection and manipulation are often used together, but selection may be a stand-alone task [1]. Several techniques can be used for selection and manipulation tasks.

Currently, there are three basic metaphors for the 3D interaction: virtual hand, virtual pointer and World-In-Miniature. However, the first two metaphors seem to be suitable with our teleoperation tasks.

The most common interaction technique which uses the metaphor of virtual hand is the simple virtual hand [1]. This technique is used for selection or manipulation tasks. It uses a one-to-one mapping between the virtual hand and the physical hand. Selection is made via direct "touching" of virtual objects. In general, this is done by intersecting the virtual hand with a virtual object. The Go-Go technique [11] also called arm-extension technique is based on the Simple Virtual Hand, but it introduces a non one-to-one linear mapping between the virtual hand and the physical hand. The selection of remote or small objects is very difficult with the simple virtual hand and the Go-Go techniques.

Another common technique is the Ray-casting [7]. This technique uses the metaphor of a laser pointer - an infinite ray extending from the virtual hand. The first object intersected along the ray is eligible for selection. This technique is very efficient to achieve selection tasks. The flash light technique [5] is based on the same principle that the Ray-casting technique, but it replaces the laser pointer by an infinite cone. It allows the selection of remote or small objects, but it presents ambiguities in the selection of close objects.

\section{DESCRIPTION OF THE FOLLOW-ME TECHNIQUE}

\section{A. Main Properties}

The FOLLOW-ME [8] is a 3D interaction technique which is used in order to fasten the selection task for teleoperation systems. It has two main characteristics:

- the VE is divided into three zones in which the interaction has its own granularity: the free manipulation zone, the scaled manipulation zone and the precise manipulation zone;

- In the precise manipulation zone, virtual guides are used to handle both precision and security of manipulation (for a review, one may refer to [9] [10] [13]).

The virtual objects may be selected by using a virtual tools (virtual pointer or virtual hand). A set of states is associated to the virtual tool whether it is in the free manipulation zone (Free_Manip_Stat), the scaled manipulation zone (Scaled_Manip_Stat) or the precise manipulation zone [Precise_Manip_Stat) of an object. Each state is linked with a specific granularity of interaction (depending on where the virtual tool is in VE):

- the Free_Manip_Stat is linked with a high granularity of interaction. This means that a movement in the real world is translated as is for the virtual tool in $\mathrm{VE}$ (gain equal or greater than 1);

- the Scaled_Manip_Stat is linked with a medium granularity of interaction. This means that a huge movement in the real world is translated into a smaller movement of the virtual tool in VE (gain comprised between 0 and 1);

- the Precise_Manip_Stat is linked with a low granularity of interaction. We use virtual guides in order to assist user. In this case, the degree of freedom for the virtual tool in VE is reduced to 1 so that the movements of the user are interpreted as go forward to the target decision or go backward decision. This means that the virtual tool may move along a $1 \mathrm{D}$ curve in VE.

A transition between one state to another corresponds to a modification of the granularity of interaction. We will see in par. III-C that it corresponds to a modification of the equation that handles the evolution of the virtual position and orientation of the virtual tool.

\section{B. Notation}

The 3D position of the user's hand is noted $\vec{P}_{r}$ and its orientation is $\overrightarrow{\theta_{r}}$ at each time $t$. The resulting virtual position in $\mathrm{VE}$ is $\overrightarrow{P_{v}}$ and the resulting orientation in $\mathrm{VE}$ is $\overrightarrow{\theta_{v}}$. The linear velocity of the hand is noted $\overrightarrow{\dot{P}_{r}}$ and its angular velocity is noted $\overrightarrow{\theta_{r}}$. The linear velocity of the virtual tool is noted $\overrightarrow{\dot{P}} v$ and its angular velocity is noted $\overrightarrow{\dot{\theta}_{v}}$.

We depict $\overrightarrow{C_{*}}$ and $\overrightarrow{\dot{\theta}}$ as the position and the orientation of 
the normal of the target to be reached.

The time at which a transition between two states happen is noted $t_{0}$.

\section{Formalisation of the FOLLOW-ME model}

For each state of the virtual tool, there exists a unique set of equations that gives its position $\vec{P}_{v}(t)$ and orientation $\overrightarrow{\theta_{v}}(t)$ in VE at time $t$. In the following, we will have a look at these equations.

These equations may have a priori free parameters. However, when the virtual tool quits at time $t_{0}$ a zone of $\mathrm{VE}$ to enter another one which has a different granularity of interaction, a state transition happens in the state graph [8] and the set of equations that compute $\overrightarrow{P_{v}}(t)$ and $\overrightarrow{\theta_{v}}(t)$ change. It is necessary that there exists a continuity constraint at time $t_{0}$ over $\overrightarrow{P_{v}}(t)$ and $\overrightarrow{\theta_{v}}(t)$. Hence, this continuity constraint permits to fix some parameters because it is possible to known the values of $\overrightarrow{P_{v}}\left(t_{0}\right), \overrightarrow{P_{r}}\left(t_{0}\right), \overrightarrow{\theta_{r}}\left(t_{0}\right)$ and $\overrightarrow{\theta_{r}}\left(t_{0}\right)$ at time $t_{0}$.

In the following, when we will type $\vec{P}_{r}\left(t_{0}\right)$ or $\overrightarrow{\theta_{r}}\left(t_{0}\right)$, it will mean: $\vec{P}_{r}\left(t_{0}\right)$ or $\overrightarrow{\theta_{r}}\left(t_{0}\right)$ computed with the former set of equations valid just before $t_{0}$ (former granularity of interaction).

Let us describe the equations giving $\overrightarrow{P_{v}}(t)$ and $\overrightarrow{\theta_{v}}(t)$ for the three states of the virtual tool.

\section{Free_Manip_Stat state}

For the Free_Manip_Stat state, we have chosen the equations associated to the Go-Go technique (see [11]) and the Ray-casting technique (see [7]).

\section{Scaled_Manip_Stat state}

For the Scaled_Manip_Stat state, we consider a gain $k_{S}$ between the real and virtual velocities. $k_{S}$ is a positive real value. As we want to be more precise in the scaled manipulation zone, $k_{S}$ must be less than 1 . We have the following set of equations:

$$
\left\{\begin{aligned}
\overrightarrow{P_{v}}(t) & =k_{S} \vec{P}_{r}(t)+\vec{P}_{v}\left(t_{0}\right)-k_{S} \vec{P}_{r}\left(t_{0}\right) \\
\overrightarrow{\theta_{v}}(t) & =\overrightarrow{\theta_{r}}(t)+\overrightarrow{\theta_{v}}\left(t_{0}\right)-\overrightarrow{\theta_{r}}\left(t_{0}\right)
\end{aligned}\right.
$$

\section{Precise_Manip_Stat state}

For the Precise_Manip_Stat state, the idea we have is to diminish the linear velocity of the virtual tool when approaching to the target so that the virtual tool position converges to $\vec{C}_{*}$. It may be written as follows:

$$
\overrightarrow{\dot{P}_{v}}(t)=k_{M} \cdot\left(\overrightarrow{C_{*}}-\vec{P}_{v}(t)\right)
$$

Where $k_{M}$ is a real parameter. In the following, we will first consider that $k_{M}$ is constant over time.
The equation 2 is a first order linear differential equation over $\vec{P}_{v}(t)$. Using the continuity constraint, the solution of this equation is:

$$
\overrightarrow{P_{v}}(t)=\left(\overrightarrow{P_{v}}\left(t_{0}\right)-\vec{C}_{*}\right) \cdot e^{\left(-k_{M} \cdot\left(t-t_{0}\right)\right)}+\vec{C}_{*}
$$

If $k_{M}>0, \overrightarrow{P_{v}}(t)$ converges to $\overrightarrow{C_{*}}$ as $t$ grows, whereas if $k_{M}<0, \vec{P}_{v}(t)$ diverges from $\vec{C}_{*}$ as $t$ grows. Besides, if $k_{M}=0$, the virtual tool keeps still $\left(\vec{P}_{v}(t)=\vec{P}_{v}\left(t_{0}\right)\right)$.

In the same way, the orientation of the virtual tool evolves as its position over time. The orientation will be given by the following equation:

$$
\overrightarrow{\theta_{v}}(t)=\left(\overrightarrow{\theta_{v}}\left(t_{0}\right)-\overrightarrow{\theta_{*}}\right) \cdot e^{\left(-k_{\theta} \cdot\left(t-t_{0}\right)\right)}+\overrightarrow{\theta_{*}}
$$

Where $k_{\theta}$ is a positive real.

The value of $k_{M}$ and $k_{\theta}$ are fixed given the information of $P_{r}$ (if a forward movement of the user's arm is detected, $k_{M}$ and $k_{\theta}$ will be fixed as positive values).

\section{EXPERIMENTS}

\section{A. Introduction}

In this paper, we show two kind of experiments. In a first stage (validation experiments), we want to measure the benefits of the use of the FOLLOW-ME technique for different conditions in a VE with the Go-Go and Ray Casting techniques. The analyzed variables are the depth of the target and the dynamic or static status of the target. The underlying question is: in which case, the FOLLOW-ME technique is really helpful? In a second stage (ARITI experiments), after having analyzed the former results, we have implemented the FOLLOW-ME technique for the tele-robotic ARITI system [10]. In particular, we have compared two designs of the ARITI system: classical ARITI system and FOLLOW-ME ARITI system.

\section{B. Aim of the validation experiments}

To show the contribution of the FOLLOW-ME technique for the assistance to other classical techniques, we have compared the classical Go-Go technique with the Go-Go technique assisted by the FOLLOW-ME technique, and on the other hand, we have compared the classical Ray-casting technique with the Ray-casting technique assisted by the FOLLOW-ME technique.

The different techniques are implemented in the same Virtual Environment which is composed of different targets placed at different depths. The targets can be static or dynamic. We think that the FOLLOW-ME technique might be particularly useful in two kinds of situations: first, when targets are far, second when targets are moving.

\section{Experimental setting}

Figure 1 shows the experimental setting. The user is situated in front of a workbench $(3,20 \times 2,40 \mathrm{~m})$. He interacts with the virtual world by using a Flystick which position $\vec{P}_{r}=$ $\left[X_{r}, Y_{r}, Z_{r}\right]$ and orientation $\overrightarrow{\theta_{r}}=\left[\theta_{r}^{X}, \theta_{r}^{Y}, \theta_{r}^{Z}\right]$ are computed 


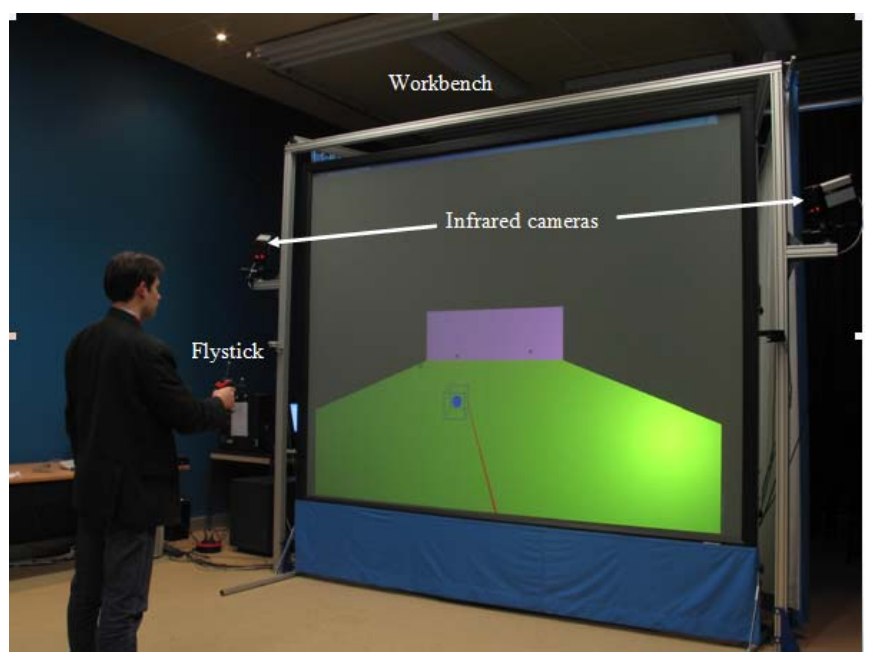

Fig. 1. Experimental setting

in real time by an ART tracking system using two infrared cameras placed on the left and the right opposite sides of the workbench.

The position and orientation of the Flystick determine the position $P_{v}=\left[X_{v}, Y_{v}, Z_{v}\right]$ and the orientation $\theta_{v}=\left[\theta_{v}^{X}, \theta_{v}^{Y}, \theta_{v}^{Z}\right]$ of the virtual tool used in the virtual world to reach the targets. The model associated with the implemented techniques gives the formal relation between the couple $\left(P_{r}, \theta_{r}\right)$ and the couple $\left(P_{v}, \theta_{v}\right)$.

\section{Experimental protocol for the validation experiments}

Four targets $T_{i}$, which are disks with the same diameter $D_{i}$, are situated at $N$ different depths in VE. The targets are green colored (see Fig.2(a)). Each target $T_{i}$ is centered on $C_{T i}=\left[X_{T i}, Y_{T i}, Z_{P i}\right]$ and situated at depth $Z=Z_{P i}$. The user may control a virtual tool which position is $\left[X_{S}, Y_{S}, Z_{S}\right]$ and orientation $\left[\theta_{S}^{X}, \theta_{S}^{Y}, \theta_{S}^{Z}\right]$. The virtual tool is represented by a virtual pointer when we apply the Ray-casting technique or by a virtual hand when we apply the Go-Go technique.

An object can be selected by intersecting it with a virtual pointer (or the virtual hand) which is activated by holding down the Flystick button.

The experiment is composed of two stages. In the first stage, the different techniques are tested on static objects. In the second case, the same techniques are tested on moving objects. In the two stages of the experiment, we implemented the two techniques (Go-Go and Ray-casting) with different types of assistance:

- without assistance (classical Go-Go and classical Raycasting techniques);

- visual assistance (see the box around the target in Fig. 2);

- FOLLOW-ME assistance (visual and command assistance).

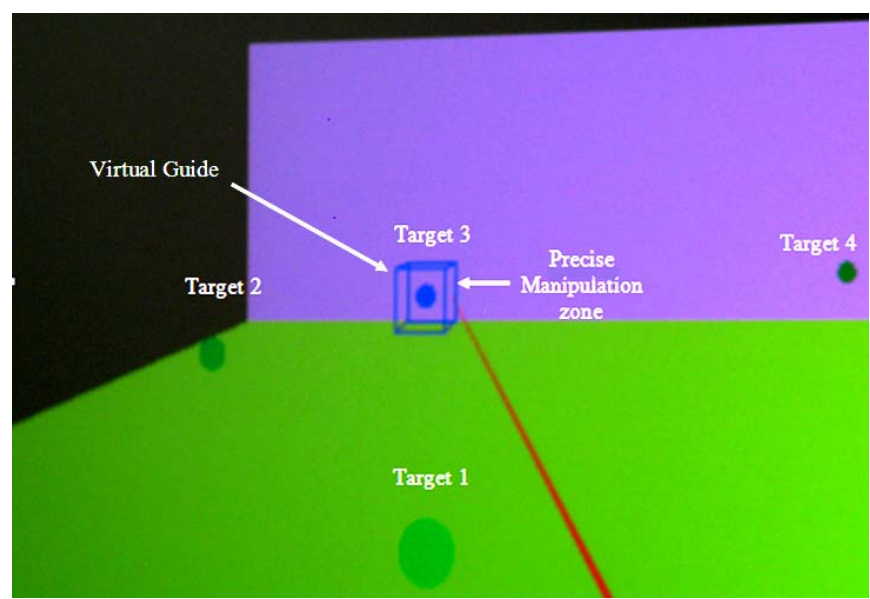

(a) The Ray-casting technique

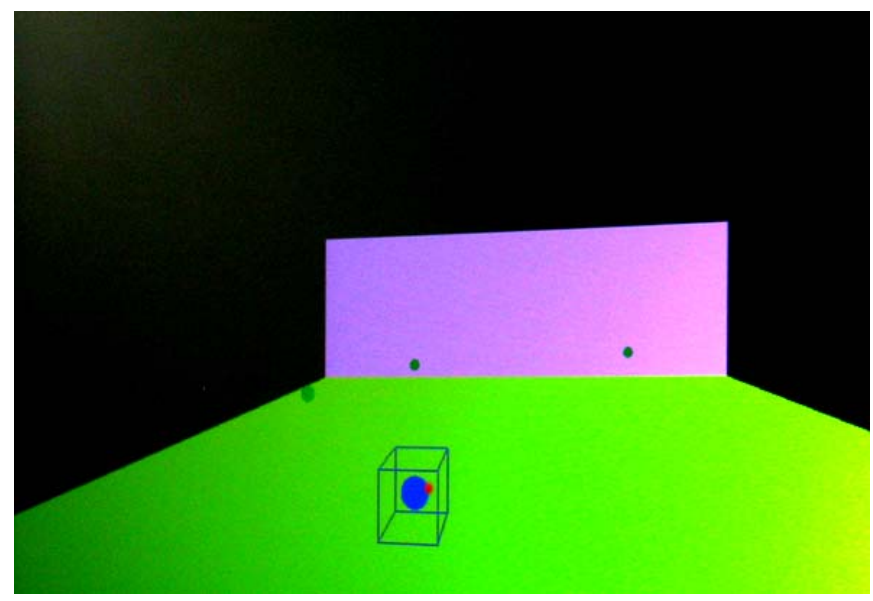

(b) The Go-Go technique

Fig. 2. Go-Go and Ray-casting techniques with the FOLLOWME assistance

The interest of implementing the two techniques with the visual assistance only (second case) is to measure the influence of the command assistance comparing to the visual cue assistance. Thus, it may be possible to show the benefits of the FOLLOW-ME technique comparing to a simple visual cue assistance.

Ten volunteers participated to the experiments. They were all male and their age varied between 25 and 35 years. Although they all had basic knowledge about computer technology, they had never experienced human-computer interaction tasks such as selection in VEs.

For each phase (static or dynamic objects), each kind of assistance (classical, visual or FOLLOW-ME assistance) and each interaction technique (Go-Go or Ray-casting techniques), the user executes a series of fifteen trials. A trial consists on selecting a predefined target (color blue) in the VE with the virtual tool with a minimum laps of time. At the beginning of each trial, the target which must be selected by the user is given randomly by the system. If a user could not select a given target after 45 seconds, the trial is considered to be a 


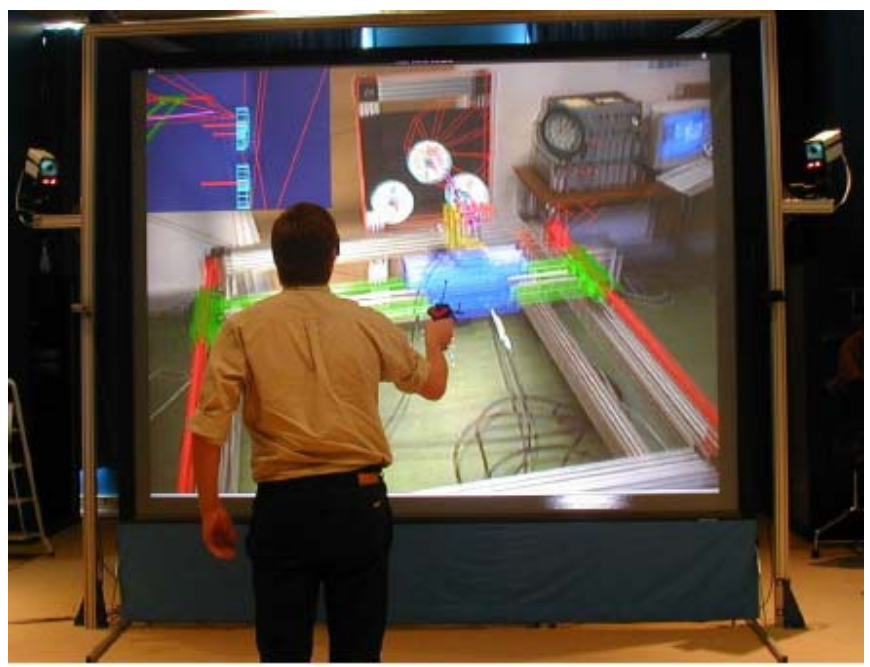

Fig. 3. Experimental setting for the ARITI framework controlling a 4-dof remote robot. 3 disk shaped targets may be selected and manipulated.

failure.

\section{E. Performance cues}

Two performance cues are considered:

- $\left(K_{1}\right)$ laps of time to reach the targets;

- $\left(K_{2}\right)$ (resp. $\left(K_{3}\right)$ ) evolution of the distance (resp. angular distance) between the virtual tool (virtual pointer or virtual hand) and the target during time.

Whereas the first cue is classically used, we think that the other ones are particularly relevant. Indeed, when far from the target, the user will probably make the virtual pointer (virtual hand) nearer to the target very easily without any assistance (visual or other). But, when approaching to the target, errors in piloting the virtual tool may occur if the target is small enough. The direct consequence of an error is the growth of $K_{2}$ or $K_{3}$ during the trial. The zone around the target in which $K_{2}$ or $K_{3}$ may grow corresponds precisely to the zone in which the user needs assistance to reach the target.

\section{F. Experimental protocol for the ARITI experiments}

In the ARITI system, the selection task consists in picking one of the 3 cylinders with a stem which is part of a 4 dof robot (see Fig. 3). This may be related to a kind of Go-Go technique. In this experiments, we compare the performance of the Go-Go technique (pick a cylinder among three with the stem without any assistance) and the Go-Go technique assisted with FOLLOW-ME. As for the former experiments (see par. IV-D), a user performs 10 trials for each of the two techniques. Each of the three cylinders may be a target to reach by the stem. The performance cues remain unchanged (see par. IV-E).

\section{RESULTS}

\section{A. Results for the validation experiments}

First, let's analyze the results for the Go-Go technique. In general, we have found that the assistance of the FOLLOWME technique gives better results than the assistance of visual cue, that itself gives better results than the classical Go-Go technique (see Fig. 4 (a),(b) and Fig. 5 (a),(b) ). In particular, if we take the example of target 3, which is far from the user, the FOLLOW-ME technique permits to drastically diminish the number of failures (timeouts). In the case of a static target 3 , the percent of timeout decreases from $16 \%$ (classical case) to $10 \%$ (visual cue only) and to less than 5\% (FOLLOW-ME) (see Fig. 5 (a)). Moreover, if the target 3 is moving, the results show a more important difference between the three kind of assistance: $27 \%$ (classical), $17 \%$ (visual cue) and less than 5\% (FOLLOW-ME) (see Fig. 5 (b)).

We may also notice that the results for the FOLLOW-ME technique for static and dynamic targets do not vary very much, whereas it is not the case for the classical and visual cue assistances. Besides, the results do not vary a lot from one target to another for the FOLLOW-ME technique. So, the first results we have tend to show that the duration of a trial is little dependent to the position of the target or the fact it is moving or not.

Second, let's analyze the results for the Ray-Casting technique. In general; we have found that the mean time to reach a target (static or dynamic) is not lessen significantly by the assistance of the FOLLOW-ME technique (see Fig. 4 (c),(d) and Fig. 5 (c),(d) ). We may explain those results with two arguments:

- First, the selection task may seem to by very easy. Even when the target is moving, the percent of timeouts is very low in for all the three kinds of assistance;

- Second, the time to automatically reach the target when entering the precise manipulation zone was non neglectable (about one second). And, looking at the duration of a trial (from four to six seconds), this delay is important.

But, one thing that the FOLLOW-ME technique seems to bring is the reproducibility of the selection task. The reproducibility is measured with the stand deviation associated to each kind of assistance. For target 1 (dynamic case) and target 3 (static and dynamic), the standard deviation of the duration of a trial is minimized by the FOLLOW-ME technique ( see Fig. 4 (d) and Fig. 5 (c),(d) ). If we look at the $K_{3}$ measure, we notice that the evolution of the angular distance to the target over time diminish more regularly for the FOLLOW-ME assistance than for the classical and visual cue assistances (see Fig. 6).

\section{B. Results for the ARITI tele-operation system}

The selection task for the ARITI teleoperation system is a particular case of the validation experiments where one of 3 cylinders, placed at the same depth, may be selected (see Fig. 3). The first results shown in the former paragraph do not indicate any benefit in using the FOLLOW-ME assistance with Ray-Casting in this case (see par. V-A). Oppositely, they tend to show that the user may be assisted efficiently by FOLLOWME if the selection technique is Go-Go. That what we wanted to test in this experiment in which one user performs 10 trials. Whereas the mean time to reach a target is $15,5 \mathrm{sec}$. (with 11,79 sec. standard deviation) for the Go-Go without 
Static Target 1: Results with the Go-Go technique

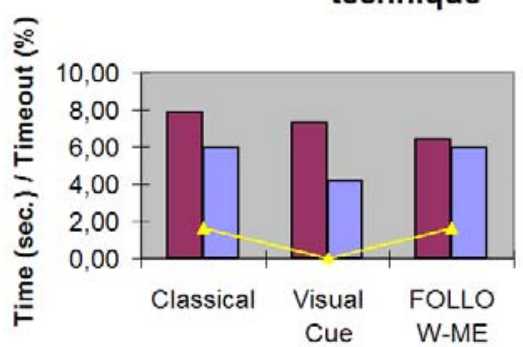

(a) Static Target 1: Go-Go technique

Static Target 1: Results with the Raycasting technique

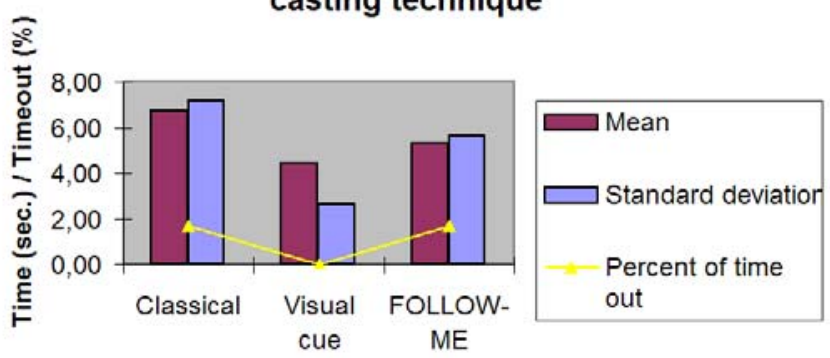

(c) Static Target 1: Ray-casting technique

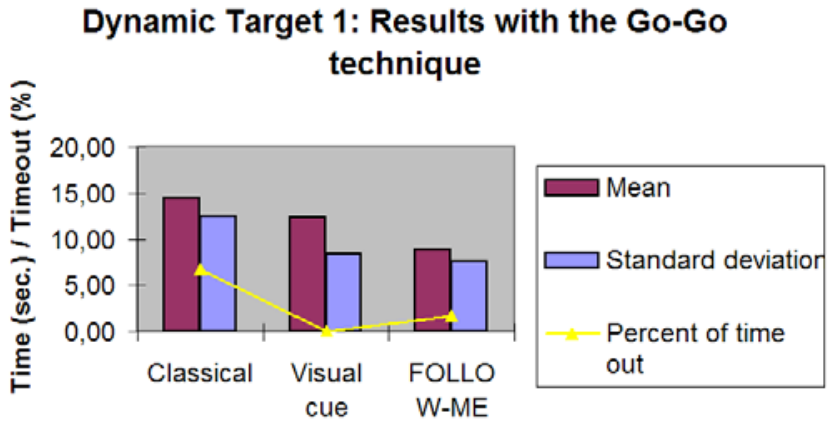

(b) Dynamic Target 1: Go-Go technique

\section{Dynamic Target 1: Results with the Ray-} casting technique

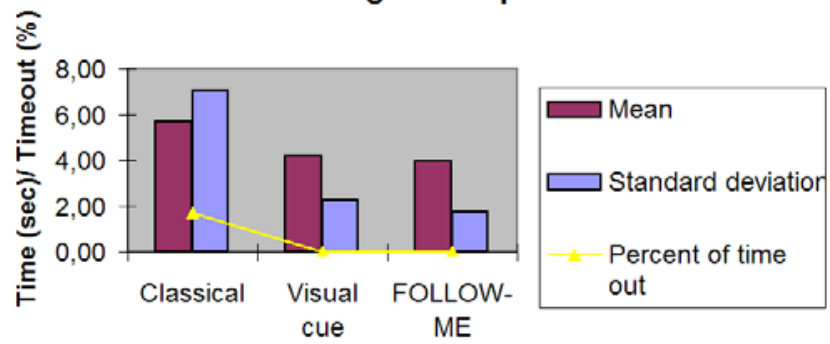

(d) Dynamic Target 1: Ray-casting technique

Fig. 4. Target 1: Trial mean duration and percent of timeout.

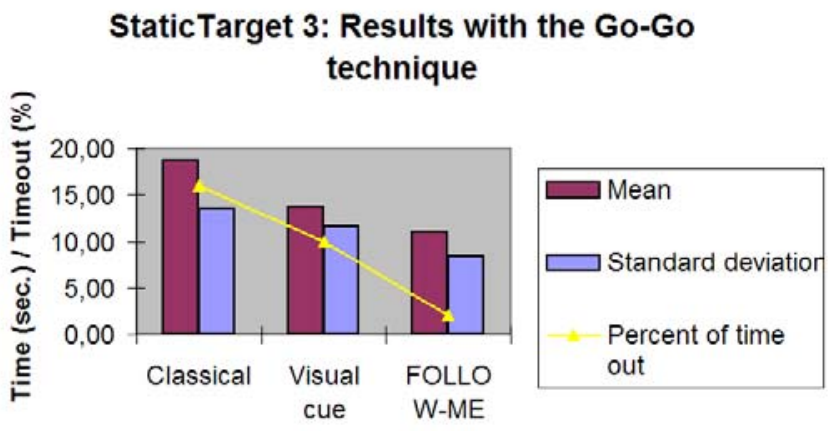

(a) Static Target 3: Go-Go technique

Static Target 3: Results with the Raycasting technique

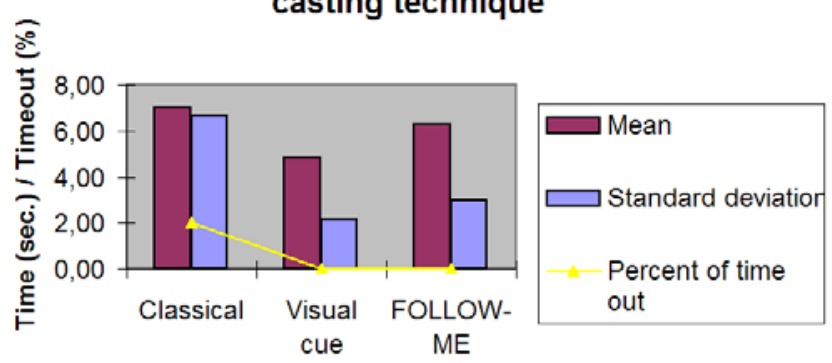

(c) Static Target 3: Ray-casting technique

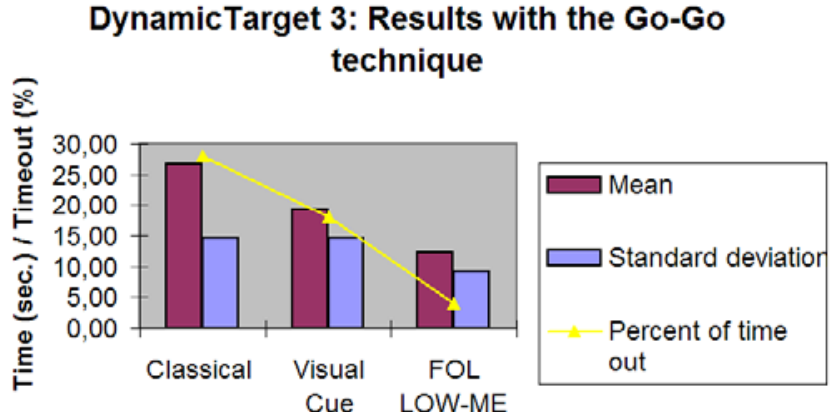

(b) Dynamic Target 3: Go-Go technique

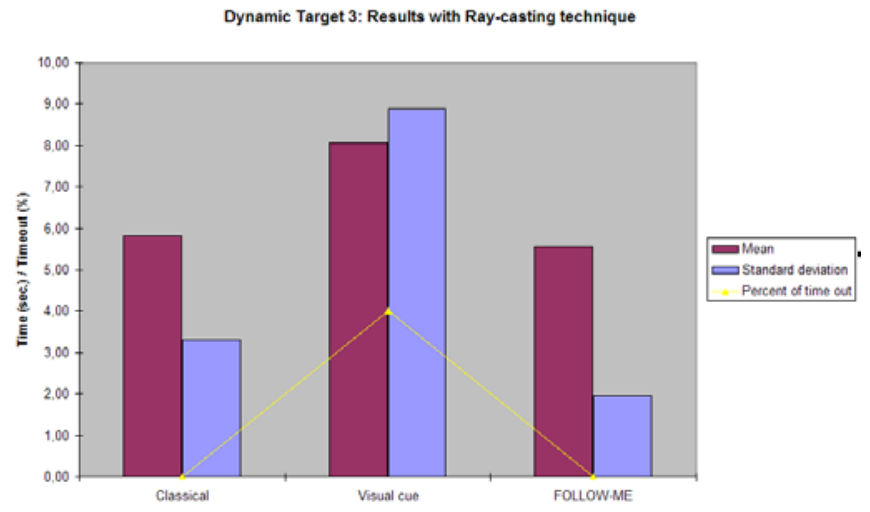

(d) Dynamic Target 3: Ray-casting technique

Fig. 5. Target 3: Trial mean duration and percent of timeout. 


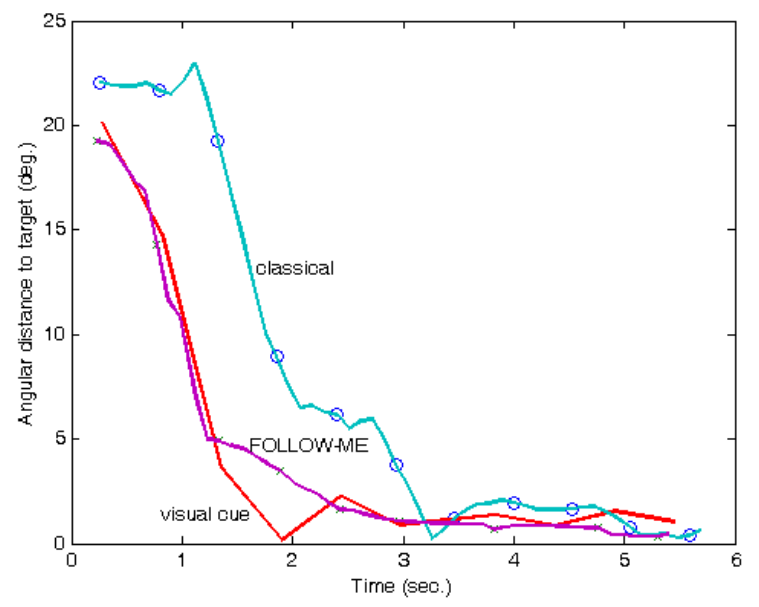

Fig. 6. Target 3 - Evolution of angular distance over time (Ray-casting, target 3 is static)

assistance, it is reduced to 3,9 sec. (with 1,81 sec. standard deviation) for the Go-Go with the FOLLOW-ME assistance.

\section{CONCLUSION}

The FOLLOW-ME technique is dedicated to assist other techniques (in this paper, we have focused on the Go-Go and Ray-casting techniques). The main characteristics of the FOLLOW-ME technique are:

- the splitting of the virtual environment into three zones (free manipulation, scaled manipulation and precise manipulation zones) differing from each other by the granularity of the interaction;

- the use of virtual guides in the precise manipulation zone.

In this article, we have first described some preliminary results about the use of FOLLOW-ME for selection tasks. They show that the FOLLOW-ME assistance permits to decrease significantly the time to reach a target for the Go-Go technique. If the target is moving, its permits to achieve the task whereas it may be very difficult without the help of FOLLOW-ME. Another important result is that the reproducibility of the selection task is facilitated by the use of the FOLLOW-ME technique, even if the mean time to reach the target is not lessen (case of the Ray-Casting technique). This may induce that the user has to concentrate less while using FOLLOWME to reach a target. So, this technique might be used with benefits for teleoperation tasks.

In a second set of experiments, we have tested the FOLLOWME assistance in the case of the ARITI teleoperation framework (see [9]). In this framework, it is very natural to implement the selection task by using the Go-Go technique. In this case, we have shown that the FOLLOW-ME assistance brings benefits for the user in terms of time to reach a target and comfort of use (less concentration is needed to perform the selection task).

\section{REFERENCES}

[1] D. Bowman, E. Kruijff, J. LaViola and I. Poupyrev, 3D user interfaces : Theory and Practice, Addison-Wesley, 2005.

[2] D. Bowman, D. Johnson and L. Hodges, Testbed evaluation of virtual environment interaction techniques, Presence: Teleoperators and Virtual Environments, 10(3):75-95, 2001.

[3] G. Burdea, and P. Coiffet, Virtual Reality technology, John Wiley et Sons, Inc Eds, New York, 1994.

[4] T. Hasegawa, T. Suehiro, T. Ogasawara, T. Matsui, K. Kitagaki and K. Takase, Model-based telerobot system manipulation skill, Proc.Int.Symp. on Advanced Robot Technology. pp. 499-506, 1991.

[5] J. Liag, JDCAD: A Highly Interactive 3D Modeling System, Computers and Graphics, 18(4):499-506, 1994.

[6] P. Milgram, S. Zhai and D. Drasic, Application of augmented reality for human-robot communication, In: IROS: Int. Conf. on Intelligent Robot and Systems. Yokohama, Japan. pp. 1467-1476, 1993

[7] M. Mine, F. Brooks and C. Sequin, Moving objects in space: exploiting proprioception in virtual environment interaction, Proceedings of SIGGRAPH'97, pp. 19-26, 1997.

[8] N. Ouramdane, F. Davesne, S. Otmane and M. Mallem, FOLLOW-ME: a new $3 D$ interaction technique based on virtual guides and granularity of interaction, ACM Internationnal Conference on Virtual Reality Continuum and Its Applcations (VRCIA 2006) pp. 137-144, Hong Kong, China, 14-17 june, 2006.

[9] S. Otmane, M. Mallem, A. Kheddar and F. Chavand, Active virtual guide as an apparatus for augmented reality based telemanipulation system on the internet, IEEE Computer Society - 33rd Annual Simulation Symposium ANSS2000, pp. 185-191, 16-20 april 2000.

[10] V. Leligeour, S. Otmane, M. Mallem Augmented Reality Interface for Free Teleoperation, International Federation of Automatic Control (PRAHA IFAC World Congress 2005), PRAHA, Czech Republic, 3-8 july 2005.

[11] I. Poupyrev, M. Billinghurst, S. Weghorst and T. Ichikawa, The Go-Go Interaction Technique: Non-Linear Mapping for Direct Manipulation in $V R$, Proceedings of the 9th annual ACM symposium on User interface software and technology, pp.79-80. ACM Press, 1996.

[12] I. Poupyrev, M. Weghost, M. Billinghnrst and T. Ichikawa Egocentric Object Manipulation Object in Virtual Environments: Empirical Evaluation of Interaction,Proceedings of Eurographics'98, 17(3), 1998.

[13] L. Rosenberg, The use of virtual fixtures to enhance telemanipulation with time delay, Proceedings, ASME Winter Annual Meeting on Haptic Interfaces for Virtual environment and Teleoperator Systems, New Orleans, Loisiana, 1993. 